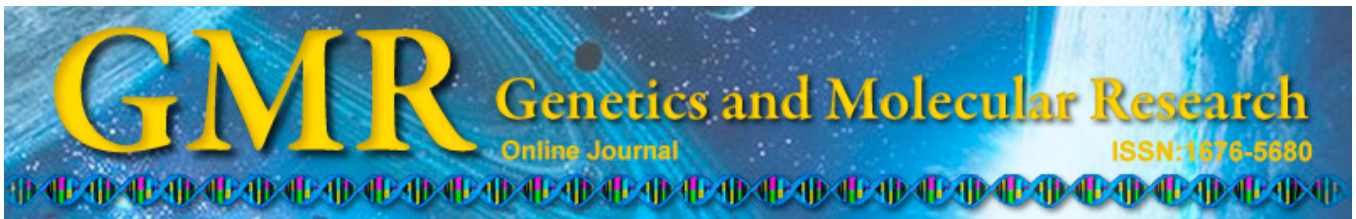

\title{
Molecular characterization, expression, and functional analysis of NOD1 in Qingyuan partridge chicken
}

\author{
Z.Y. Tao ${ }^{1,2,3}$, C.H. Zhu' ${ }^{2}$ Z.H. Shi' ${ }^{2}$ C. Song ${ }^{2}$, W.J. Xu' ${ }^{2}$, W.T. Song ${ }^{2}$, \\ J.M. Zou' ${ }^{2,3}$ and A.J. Qin ${ }^{1,3}$ \\ ${ }^{1}$ Ministry of Education Key Laboratory for Avian Preventive Medicine, \\ Yangzhou University, Yangzhou, China \\ ${ }^{2}$ Jiangsu Institute of Poultry Sciences, Yangzhou, China \\ ${ }^{3}$ Jiangsu Co-Innovation Center for Prevention and Control of Important Animal \\ Infections Diseases and Zoonoses, Yangzhou \\ Corresponding author: A.J. Qin \\ E-mail: aijian@yzu.edu.cn
}

Genet. Mol. Res. 14 (1): 2691-2701 (2015)

Received June 4, 2014

Accepted October 17, 2014

Published March 30, 2015

DOI http://dx.doi.org/10.4238/2015.March.30.29

\begin{abstract}
Nucleotide-binding oligomerization domain-containing protein-1 (NOD1) is a cytoplasmic pattern recognition receptor (PRR) and a key member of the NOD-like receptor (NLR) family. It has been reported that NLRs recognize a variety of microbial infections to induce the host innate immune response via modulation of NF- $\mathrm{KB}$ signaling. However, no reports on chicken NOD1 have been reported to date. In the current study, the full-length cDNA sequence of $N O D 1$ was cloned. The complete open reading frame of NOD1 contains $2856 \mathrm{bp}$ and encodes a 951 amino acid protein. Structurally, it is comprised of one caspase recruitment domain at the N-terminus, seven leucine-rich repeat regions at the $\mathrm{C}$-terminus, and one NACHT domain between the $\mathrm{N}$ and $\mathrm{C}$-termini. Phylogenetic analyses showed that chicken NOD1 clusters with duck and turkey. Furthermore, tissue-specific expression analyses of chicken NOD1 were performed using quantitative reverse
\end{abstract}


transcription-PCR. NOD1 is widely distributed in various tissues, with the highest expression observed in testes. Finally, induced expression of $c h N O D 1$ and its associated adaptor molecule receptor-interacting protein 2, as well as the effector molecule $N F-\kappa B$, was observed following $S$. enterica serovar Enteritidis infection. These findings highlight the important role of chicken NOD1 in response to pathogenic invasion. The present study is the first report of the cloning, expression, and functional analysis of chicken NOD1 and provides the foundation for future research on the structure and function of chicken NOD1.

Key words: Chicken; Receptor-interacting protein 2; Full-length cDNA; Nucleotide-binding oligomerization domain-containing protein $-1 ; N F-\kappa B$

\section{INTRODUCTION}

Four pattern recognition receptor families have been discovered in animals: Tolllike receptors (TLRs), retinoic acid-induced gene-like receptors (RLRs), NOD-like receptors (NLRs), and AIM2-like receptors (ALRs) (Akira et al., 2006; Uematsu and Akira, 2008; Kawai and Akira, 2009; Hayashi et al., 2011). TLRs have been extensively studied and have been shown to recognize bacterial ligands at the plasma membrane as well as on the luminal side of intracellular vesicles (Uematsu and Akira, 2006; Masumoto et al., 2006). Discovered more recently are the NLRs which are located in the cytosol and are intracellular pattern recognition receptors (PRRs) (Inohara et al., 2005; Ting and Davis, 2005). Similar to TLRs, they are involved in immunity against pathogenic microorganisms (Jacobs and Damania, 2012) and play an important role in the defense against pathogen infection by recognizing pathogenassociated molecular patterns (PAMPs). As well, they are involved in initiating the innate immune response, thereby leading to an adaptive immune response (Kawai and Akira, 2009; Kersse et al., 2011; Koizumi et al., 2012).

Structurally, the NLR family of receptors are large multi-domain proteins typically characterized by a tripartite architecture: an N-terminal protein-protein interaction domain composed of a caspase activation and recruitment domain (CARD), pyrin domain (PYD), or baculovirus "inhibitor of apoptosis" repeat (BIR) domain that generates and transmits downstream signals, a central nucleotide oligomerization (NACHT) domain that mediates selfregulation and oligomerization, and a C-terminal leucine-rich repeat that recognizes specific ligands for receptor association (Werts et al., 2006; Wilmanski et al., 2008). NOD1 and NOD2 were first discovered as cytosolic proteins involved in the intracellular recognition of microbes and their products (Philpott and Girardin, 2010). NOD1 senses meso-diaminopimelic acid (meso-DAP)-containing peptidoglycans of Gram-negative bacteria (Chamaillard et al., 2003; Girardin et al., 2003), whereas NOD2 detects muramyl dipeptide (MDP), the largest peptidoglycan motif that is common to Gram-negative and -positive bacteria (Inohara et al., 2000; Girardin et al., 2001).

It has been shown that NOD1 and NOD2 are linked to host defenses against various pathogenic bacteria, including Listeria monocytogenes (Opitz et al., 2006), Pseudomonas aeruginosa (Travassos et al., 2005), Staphylococcus aureus (Hruz et al., 2009), and Salmonella enterica serovar Typhimurium (Geddes et al., 2010). Studies have demonstrated that RIP2/ CARDIAK/RIPK2 (RICK) is a critical downstream mediator of NOD1 and NOD2 signaling 
(Inohara et al., 1999). Additionally, it has been shown that NOD1/NOD2 signaling is involved in NF- $\mathrm{KB}$ activation and intestinal inflammation in vivo and in vitro (Geddes et al., 2010; Keestra et al., 2011; Oehlers et al., 2011). However, the role of NOD1/NOD2 signaling in birds remains unclear.

Comparative genomic and sequence analyses found NOD1 orthologs in all vertebrate species analyzed, whereas NOD2 was absent from the genomes of avian, reptilian, and amphibian species (Boyle et al., 2013). Chicken has been a model system in embryology for more than a century (Stern, 2005). Thus, the purpose of the present study was to clone the full-length cDNA of the chicken NOD1 gene, analyze its expression profile in various chicken tissues, and evaluate the gene expression of NOD1, RIP2, and NF-kB after Salmonella infection. Our novel findings provide significant scientific value for studying the function of NOD1 in birds.

\section{MATERIAL AND METHODS}

\section{Animals and treatment}

Healthy Qingyuan partridge chickens were obtained from the National Chicken Genetic Resources (Jiangsu, China). The standard Salmonella enteritidis strain 50336 was obtained from the College of Veterinary Medicine of Yangzhou University (Yangzhou, China). The Qingyuan partridge chicken treatment group was subcutaneously injected with $10^{8} \mathrm{CFU} /$ $\mathrm{mL}(0.2 \mathrm{~mL})$ of Salmonella. The negative control group was injected with saline $(0.2 \mathrm{~mL})$. Spleen samples were collected after 1, 3,5, and 7 days. Ten chickens ( 5 males, 5 females) were randomly selected at each time point.

\section{RT-PCR and rapid amplification of cDNA ends (RACE)}

The RACE technique was used to obtain the full-length chNOD1 cDNA sequence, including the 5'- and 3'-untranslated regions (UTRs). Briefly, a PCR fragment of NOD1 was obtained from Qingyuan partridge chicken-specific complimentary DNA (cDNA) using Taq Polymerase and primers designed based on the predicted reference sequence of NOD1 (XM418777.2). The primers are designated NOD1-F1 and NOD1-R1, and the fragment length was $422 \mathrm{bp}$. The primers $5^{\prime}-\mathrm{GSP} 1$ and $3^{\prime}$-CDS primer A were used in $5^{\prime}$ and $3^{\prime}$ first strand cDNA synthesis, respectively. Four specific nested primers, 5'-NOD1-GSP2, 5'-NOD1-GSP3, 3'-NOD1-GSP1', and 3'-NOD1-GSP2', were designed according to the known chNOD1 cDNA fragment sequence. 5'-AAP, 5'-AUAP, and Universal Primer A mix (UPA) were obtained from the corresponding RACE Kits. 3'-RACE was conducted with the SMART RACE cDNA Amplification kit (Clontech) and 5'-RACE was conducted with 5'-RACE System for Rapid Amplification of cDNA Ends (version 2.0) kit (Invitrogen) using $10 \mathrm{mg}$ of RNA isolated from the spleen, according to manufacturer instructions.

The deduced full-length chNOD1 sequence was assembled using the 3'- and 5'-RACE sequences and the DNAMAN software (Lynnon BioSoft). To confirm the complete sequence of the open reading frame (ORF), an ORF primer pair (NOD1-ORF-F and NOD1-ORF-R) was designed according to the assembled full-length chNOD1 cDNA. All PCR products were gel-purified, cloned into the pMD18T vector (Takara Biotechnology Co., Dalian, China), and commercially sequenced. 


\section{Predicted amino acid sequence of $\operatorname{chNOD1}$}

Amino acid sequence analyses and structural predictions of $\operatorname{chNOD1}$ were performed using various bioinformatic software tools. The online analysis tool ORF Finder (http://www. ncbi.nlm.nih.gov/projects/gorf/orfig.cgi) was used to analyze the predicted chNOD1 amino acid sequence. Analyses of physical and chemical parameters were performed using ExPASYProtParam (http://expasy.org/tools/ protparam.html). Transmembrane protein structure prediction was conducted using TMPRED (http://www. ch.embnet.org/software/TMPRED_form. html). Signal peptide prediction was performed using the SignalP 4.1 Server (http://www. cbs.dtu.dk/services/SignalP/). Protein secondary structure prediction was conducted using SOPMA (http://npsa-pbil.ibcp.fr/cgi-bin/secpred_sopma.pl). We used the Simple Modular Architecture Research Tool (http://smart.embl-heidelberg.de) to analyze the protein motifs of chNOD1. The neighbor-joining phylogenetic tree was constructed using MEGA 4.0 (http:// www.megasoftware.net/mege).

\section{RNA isolation}

Total RNA was isolated from tissue samples using the TRNzol-A $^{+}$reagent (TIANGEN, China) according to the manufacturer protocol. Extracted RNA was dissolved in RNase-free water and quantified using an UV visible range spectrophotometer at an optical density of $260 \mathrm{~nm}$. RNA was treated with DNase (TaKaRa, Shanghai, China) following the manufacturer protocol. The integrity of RNA aliquots were verified using electrophoresis on a $1 \%$ agarose gel stained with ethidium bromide. Reverse transcription (RT) reactions $(20 \mu \mathrm{L})$ consisted of: $1.0 \mu \mathrm{g}$ total RNA, 50 units Superscript II reverse transcriptase (Invitrogen/Life Technologies), 40 units of RNase inhibitor (Invitrogen/Life Technologies), $0.5 \mathrm{mM}$ dNTPs, and $100 \mathrm{ng}$ oligo(dT) primer. cDNA products were stored at $-20{ }^{\circ} \mathrm{C}$ until quantitative PCR analyses.

\section{mRNA quantification using qRT-PCR}

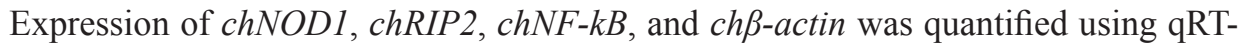
PCR and the Stratagene Mx3000P qPCR system (Agilent Technologies Inc., USA). Primer sequences are shown in Table 1. PCR reactions were performed in $20 \mu \mathrm{L}$ and consisted of $2 \mu \mathrm{L}$ (100 ng) cDNA, $10 \mu \mathrm{L}$ of SYBR real-time PCR premix (2X) (Tiangen), $0.4 \mu \mathrm{L}$ of ROX reference dye (50X), $0.8 \mu \mathrm{L}$ of each primer $(10 \mathrm{mM})$, and $6.8 \mu \mathrm{L}$ of $\mathrm{ddH}_{2} \mathrm{O}$. All qRT-PCR reactions were performed in triplicate.

\section{Statistical Analyses}

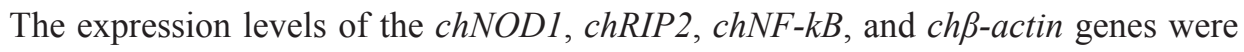
analyzed using the $2^{-\Delta \Delta \mathrm{Ct}}$ method (Pfaffl, 2001), where the Ct value represents the cycle number at which the fluorescence intensity trace of each reaction intersects the threshold line. The specific formulas were as follows: $\Delta \mathrm{Ct}=\mathrm{Ct}_{N O D I}-\mathrm{Ct}_{\beta \text {-actin }}, \Delta \Delta \mathrm{Ct}=\Delta \mathrm{Ct}_{\text {sample }}-\Delta \mathrm{Ct}_{\text {calibrator }}$. Results are shown as fold-change determined using the $2^{-\Delta \Delta \mathrm{Ct}}$ method. 
Table 1. Primers used in the study.

\begin{tabular}{|c|c|c|}
\hline Primer name & Primer sequence & Primer purpose \\
\hline NOD1-F1 & 5'-TATTTA TGTCTTTGGCGATGC-3' & \multirow[t]{4}{*}{ 422-bp fragment PCR } \\
\hline NOD1-R1 & 5'-CTCAGTTCCTGTCCGTCCT-3' & \\
\hline 5'-GSP1 & 5'-ACGGCTTCATCTTCCT-3' & \\
\hline 5'-NOD1-GSP2 & 5'-GAAAAACTTGGCCCCTATATCC-3' & \\
\hline 5'-NOD1-GSP3 & 5'-AAGACTTTGTATCGTTTGCAGC-3' & \multirow[t]{4}{*}{ 5' RACE PCR } \\
\hline 5'-AAP & 5'-GGCCACG CGTCGACTAGTACGGGIIGGGIIGGGIIG-3' & \\
\hline 5'-AUAP & 5'-GGCCAC GCGTCGACTAGT AC-3' & \\
\hline 3'- CDS primer A & $\begin{array}{l}\text { 5'-AAGCAGTGGTATCAACGCAGACTAC(T) } 30 \text { V N-3' } \\
(\mathrm{N}=\mathrm{A}, \mathrm{C}, \mathrm{G} \text {, or } \mathrm{T} ; \mathrm{V}=\mathrm{A}, \mathrm{G}, \text { or } \mathrm{C})\end{array}$ & \\
\hline 3'-NOD1-GSP1' & 5'-TTGCTACCCAGATCAGGACCCCACAGA-3' & \multirow{5}{*}{ 3' RACE PCR } \\
\hline 3'-NOD1-GSP2' & 5'-ACCCTCTGGTGCTGTTGGTTAGCCTTC-3' & \\
\hline Universal Primer A & Long: 5'-CTAATACGACTCACT ATAGGGCA & \\
\hline $\operatorname{mix}(\mathrm{UPA})$ & AGCAGTGGTATCAACGCAGAGT-3' & \\
\hline & Short: 5'- CTAATACGACTCACTATAGGGC-3' & \\
\hline NOD1-ORF-F & 5'-CTTCTTGCTGGCTCA GCTAT GG-3' & \\
\hline NODI-ORF-R & 5'-CTCA GAAGCAAATGATCCGTTC-3' & \\
\hline NOD1-F & 5'-GCGATGCAGGAATTGGAAAA-3' & \multirow{2}{*}{ qRT-PCR } \\
\hline NODI-R & 5'-TGTGAAAAGAACCGTA TGAGGGA-3' & \\
\hline RIP2-F & 5'-TGGGAGTGAACTATTTG-3' & \multirow[t]{2}{*}{ qRT-PCR } \\
\hline RIP2-R & 5'-GTTTTCTGACTGGGATT-3' & \\
\hline$N F-k B-\mathrm{F}$ & 5'-GTAACTCGGACAGGGCAG-3' & \multirow{2}{*}{ qRT-PCR } \\
\hline$N F-k B-\mathrm{R}$ & 5'-CGTATCGGAATCGAAAAC-3' & \\
\hline$\beta$-actin-F & 5'-TGCTGTGTTCCCATCTATCG-3' & \multirow[t]{2}{*}{ qRT-PCR } \\
\hline$\beta$-actin-R & 5'-TTGGTGACAATACCGTGTTCA-3' & \\
\hline
\end{tabular}

\section{RESULTS}

\section{Cloning and sequence analyses of $\operatorname{chNOD1}$}

A 422-bp fragment was amplified using the primers NOD1-F1 and NOD1-R1. Based on the sequence information, a 750-bp fragment from the $5^{\prime}$ end and a 3400-bp fragment from the 3' end were obtained after 5'- and 3'-RACE, respectively. The full-length chNOD1 cDNA (4502 bp) was obtained using the DNAMAN software, and its sequence has been submitted to GenBank (accession number: JX465487). The cDNA contained a 2856-bp ORF, a 124-bp 5' UTR, and a 1497-bp 3' UTR followed by a 22 bp poly(A) tail. BLASTn was used to obtain chNOD1 and chicken genomic sequences. chNOD1 is located on chromosome 2.

\section{Analysis of the predicted chNOD1 amino acid sequence}

\section{Physical and chemical properties of chNOD1}

Analyses of the predicted chNOD1 amino acid sequence indicated that the ORF encodes a putative 951 amino acid protein with a predicted molecular weight of 108,676.2 $\mathrm{Da}$ and a theoretical isoelectric point (PI) of 6.58. The instability index (II) is calculated to be 36.77 , thus classifying the NOD1 protein as stable. The grand average of hydropathicity (GRAVY) is -0.160 , indicating it is a hydrophilic protein.

\section{Prediction of signal peptides and transmembrane helices in chNOD1}

A signal peptide prediction was performed using Signal 4.1; however, no signal pep- 
tides were found in chNOD1. Transmembrane protein structure prediction was performed using TMHMM 2.0, but no predicted transmembrane protein was found in chNOD1 (data not shown).

\section{Predicted secondary structure prediction of chNOD1}

Alpha helices $(\mathrm{H})$, extended strands $(\mathrm{E})$, beta turns $(\mathrm{T})$ and random coils $(\mathrm{L})$ are the four basic secondary structure elements of protein. The secondary structure of chNOD1 was analyzed using SOPMA. Prediction analyses revealed that chNOD1 consists of 527 alpha helices $(55.42 \%), 95$ extended strands $(9.99 \%), 43$ beta turns $(4.52 \%)$, and 286 random coils $(30.07 \%)$. This indicated that chNOD1 secondary structure is dominated by alpha helices and random coils.

\section{Motif and sequence homology analyses of the chNOD1 protein}

In this study, analyses of chNOD1 motifs revealed the presence of a CARD and NACHT [neuronal apoptosis inhibitory protein (NAIP), Class II transactivator (CIITA), HET$\mathrm{E}$, and TP-1] domain, as well as a distinctive arrangement of seven leucine-rich repeat regions. These findings are similar to duck, turkey, human, mouse, and fish NOD1 (Figure 1). A low complexity region exists in chicken, duck, and turkey that is smaller than the region in human, mouse, or fish. In addition, the structural analyses confirmed that chNOD1 belongs to the NOD-like receptor family.

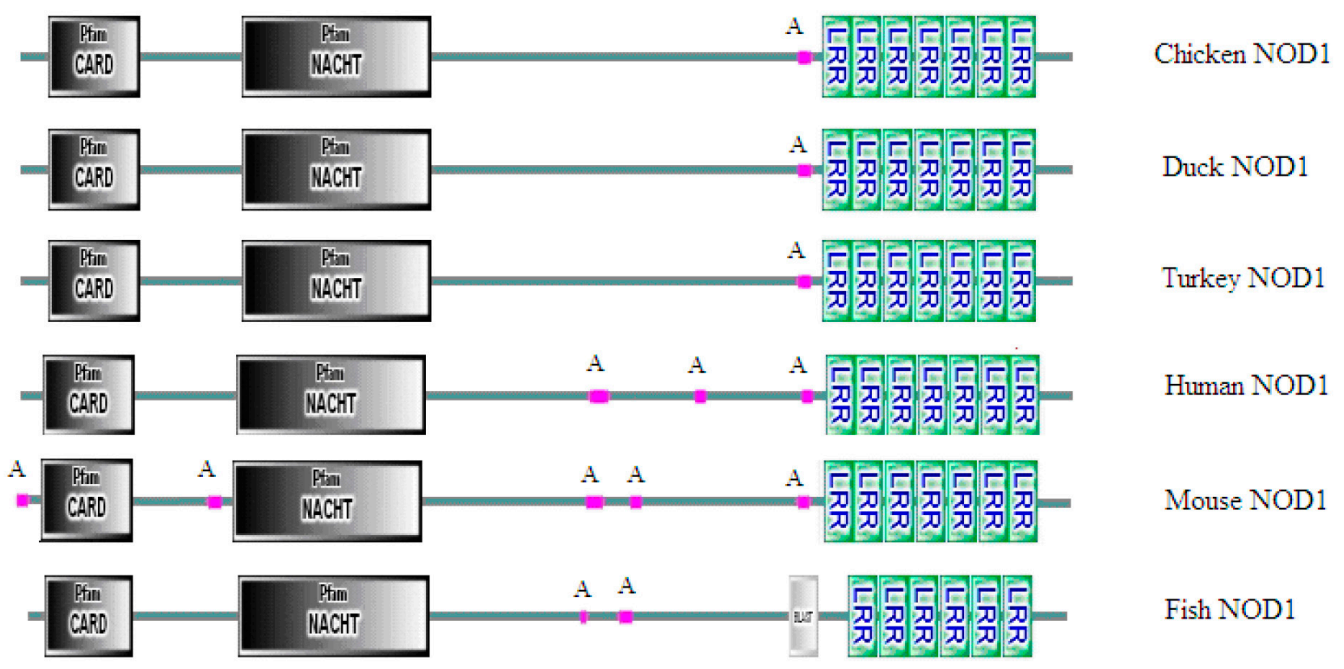

Figure 1. Comparison of the protein motifs in chicken NOD1, duck NOD1, turkey NOD1, human NOD1, mouse NOD1, and fish NOD1. CARD: Caspase recruitment domain; NACHT: (NAIP, CIITA, HET-E, and TP-1 containing domain); leucine-rich repeat; bar A: Low-complexity region. 
The NOD1 amino acid sequences of different species were aligned using NCBI BLASTp. BLAST analyses showed that the NOD1 protein from Qingyuan partridge chicken shared 99\% amino acid sequence similarity with the predicted chicken NOD1 protein (GenBank: XP-418777.2). chNOD1 showed 96\% identity with turkey NOD1, 91\% with duck NOD1, 61-68\% with mammalian NOD1, and 52\% with fish NOD1. A phylogenetic tree was constructed to show the clustering of NOD1 and confirmed that chNOD1 has the greatest similarity with NOD1 from duck and turkey (Figure 2).

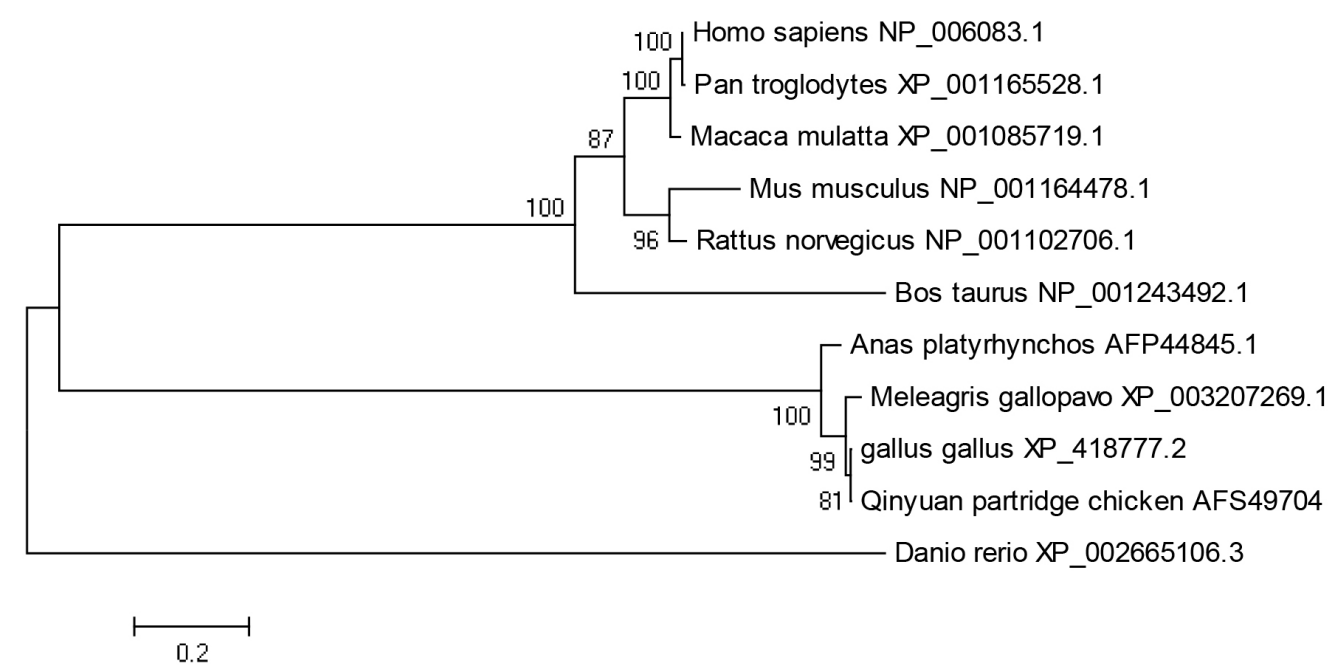

Figure 2. Consensus neighbor-joining tree based on the sequences of different types of NOD1. Sequences were aligned using the Clustal W program. The phylogenetic tree was constructed by neighbor-joining methods using MEGA5.0. Figures represent percentage values from 1000 bootstrapping trials. Other sequences were selected from GenBank.

\section{Expression levels of $\operatorname{ch}$ NOD1 mRNA expression in tissues}

chNOD1 mRNA expression was detected in 12 tissues from 12-week-old Qingyuan partridge chickens ( 5 males, 5 females). Statistical analyses were applied to evaluate any differences due to sex, however, no sex-specific effects were observed. Therefore, chNOD1 expression was analyzed using a combination of males and females. We detected varying levels of chNOD1 mRNA expression in heart, liver, spleen, lung, kidney, thymus, bursa, small intestine, oviduct, ovaries, testes, and brain. The chNOD1 was most highly expressed in testes (5.45), followed by oviduct (4.62), heart (3.87), spleen (3.19), thymus (3.02), lung (2.98), kidney (2.71), and ovary (2.44). The lowest levels of expression was observed in bursa (1.07), brain (1.38), liver (1.27), and intestine (1.0) (Figure 3). 


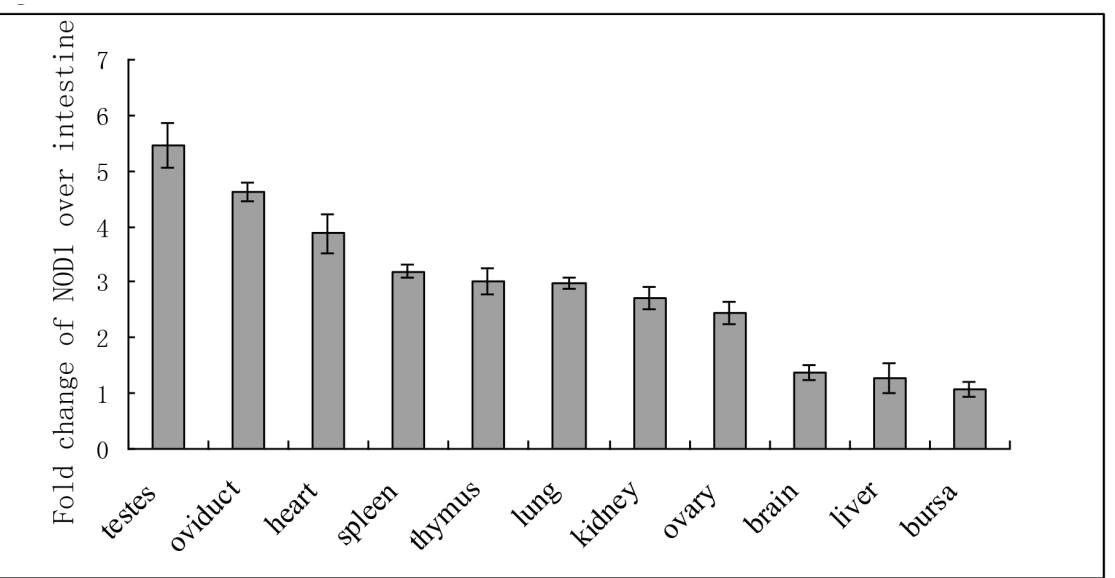

Figure 3. chNOD1 mRNA expression in various tissues in Qingyuan partridge chicken. Data are reported as means $\pm \mathrm{SE}$.

\section{Expression levels of $c h N O D 1$, chRIP2, and $c h N F-k B$ mRNA during Salmonella challenge}

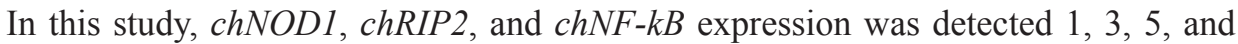
7 days after the Salmonella Enteritidis challenge in Qingyuan partridge chickens. No sex-

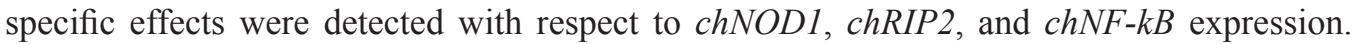

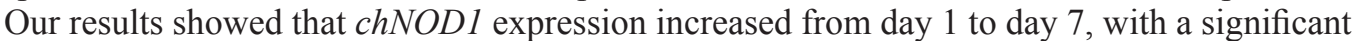
increase at day 5 . The downstream genes $c h R I P 2$ and $c h N F-k B$ were significantly increased after 3 and 5 days (Figure 4).

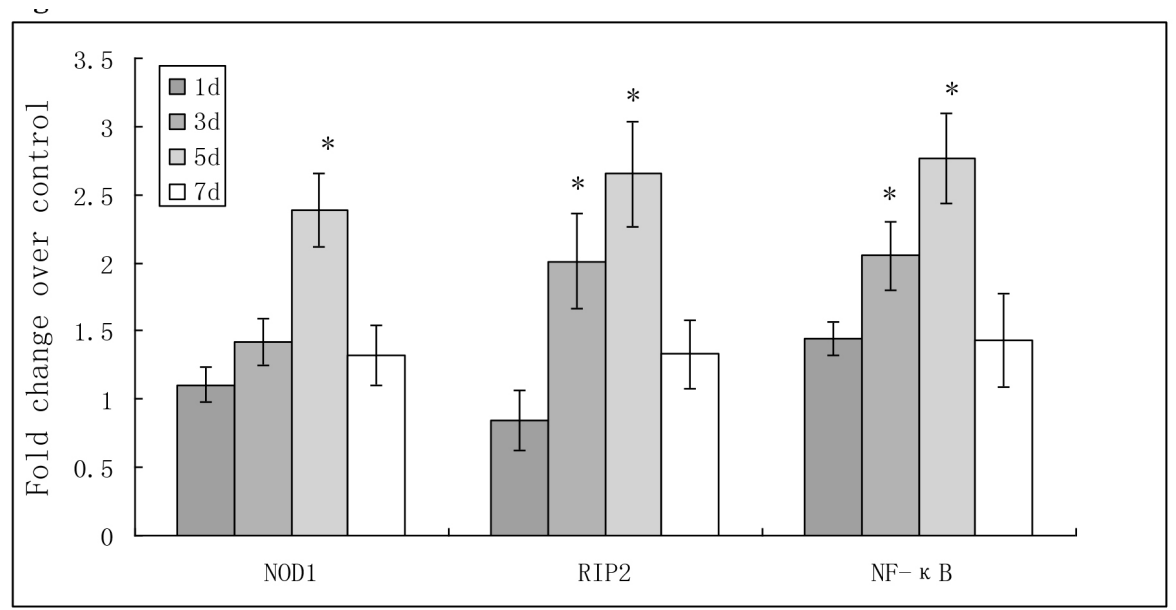

Figure 4. chNOD1, chRIP2, and $c h N F-k B$ mRNA expression during Salmonella challenge after 1, 3, 5, and 7 days. Data are reported as means \pm SE. *indicates significantly different from control. 


\section{DISCUSSION}

NOD1 gene sequences have been cloned successfully from mouse (Inohara et al., 1999), cow (Zimin et al., 2009), pig (Tohno et al., 2008), and fish (Chen et al., 2010), however, no full-length chicken NOD1 cDNA sequence had been reported. In this study, the chNOD1 gene was successfully cloned from Qingyuan partridge chicken. The Qingyuan partridge chicken NOD1 protein showed $99.8 \%$ identity with the predicted chicken NOD1 protein, however, we found two amino acid differences at residues I619T and R744K. CARD domains are interaction motifs found in a wide array of proteins, typically those involved in inflammation and apoptosis processes. A number of CARD proteins play a role in regulating inflammation in response to bacterial and viral pathogens, as well as to a variety of endogenous stress signals (Bouchier-Hayes and Martin, 2002). The NACHT domain is an evolutionarily conserved protein domain found in association with other domains, such as the CARD domain, pyrin domain, HEAT repeat domain, WD40 repeats, leucine-rich repeats or BIR repeats (Koonin and Aravind, 2000). Leucine-rich repeats are 22-28 amino acid motifs that are found in proteins with diverse functions and cellular locations. In the current study, we noted one CARD at the $\mathrm{N}$-terminus, seven leucine-rich repeats regions at the $\mathrm{C}$-terminus, and one NACHT domain between the $\mathrm{N}$ - and $\mathrm{C}$-termini. The structural composition indicates that chNOD1 may function similarly as the NOD1 proteins in other species. Phylogenetic tree analyses showed that chicken NOD1 clustered with the duck and turkey NOD1. The amino acid sequence of chNOD1 has a high degree of similarity with the mammalian and fish NOD1.

Previous studies have shown that NOD1 is widely expressed in many cell types and tissues. For example, the NOD1 transcript is expressed in various human adult tissues including heart, placenta, lung, skeletal muscle, liver, kidney, spleen, thymus, and ovary (Inohara et al., 1999). Quantitative PCR detected NOD1 mRNA in multiple tissues isolated from adult and newborn swine, including the esophagus, duodenum, jejunum, ileum, ileal Peyer's patches, colon, spleen, and mesenteric lymph nodes (Tohno et al., 2008). The expression profile showed that $g c N O D 1$ and $g c N O D 2$ were ubiquitously expressed in adult tissues in grass carp (Chen et al., 2010). However, it remained unknown whether the NOD1 gene was expressed in chicken tissues.

In this study, we detected chNOD1 transcripts in 12 tissues with varying expression levels. These results demonstrating that chNOD1 is widely expressed in chicken tissues, which is in agreement with other reports in other species. Conventional immune organs such as the thymus and spleen, express high levels of NOD1, whereas bursae express NOD1 at low levels. King et al. (2009) reported that NOD1 and NOD2 mRNAs were constitutively expressed in the endometrium. In the female reproductive tract, NOD1 gene expression was highest in the oviduct and was constitutively expressed in this tissue. Our results show the highest chNOD1 expression in the testes and oviduct. These data indicate that NOD1 may have an important role in the reproductive system.

NOD1 is a member of the NLR family containing an N-terminal CARD domain, required to trigger NF- $\mathrm{KB}$ signaling. The CARD domain of NOD1 binds the CARD domain of RIP2 through homophilic CARD-CARD interactions (Correa et al., 2012) and induces NF$\kappa B$. Several studies have shown that NOD1 detects the minimal tri-DAP structure of Gramnegative bacteria, including Salmonella typhimurium, Legionella pneumophila, and Shigella flexneri (Berrington et al., 2010; Keestra et al., 2011; Robertson and Girardin, 2013). In order to examine whether Salmonella infection activates chNOD1 signaling in chicken, Salmonella 
enteritidis was used to infect Qingyuan partridge chickens. We found increased expression of chNOD1, chRIP2, and chNF-kB after the Salmonella challenge. These results suggest that chNOD1 signaling is involved in the process of eliciting an immune response to Gramnegative bacteria. NOD2 has been shown to activate RIP2 and $N F-k B$ (Inohara et al., 2000; Girardin et al., 2001; Zhao et al., 2012). In this study, chNOD1 expression was significantly upregulated 5 days after the Salmonella challenge, however, chRIP2 and $c h N F-k B$ increased 3 and 5 days after the challenge. NOD2 is not expressed in chicken, therefore, we speculate that other genes are interacting with $R I P 2$ to mediate $N F-k B$ expression.

There are few studies of NLRs in avian species. Therefore, we cloned the full-length NOD1 cDNA, confirmed its expression in a wide range of tissues, and determined that Salmonella enteritidis activates NOD1 signaling in chicken. Our novel findings provide important information regarding the study of NODI in birds.

\section{ACKNOWLEDGMENTS}

Research supported by National Natural Science Foundation of China (\#31101795 and \#31101833); the Graduate Research and Innovation Program of Jiangsu College, China (\#CXZZ13-0916); the Priority Academic Program Development of Jiangsu Higher Education Institutions; and the Science and Technology Public Service Platform Project of Jiangsu, China (\#BM2012060).

\section{REFERENCES}

Akira S, Uematsu S and Takeuchi O (2006). Pathogen recognition and innate immunity. Cell 124: 783-801.

Berrington WR, Iyer R, Wells RD and Smith KD, et al. (2010). NOD1 and NOD2 regulation of pulmonary innate immunity to Legionella pneumophila. Eur. J. Immunol. 40: 3519-3527.

Bouchier-Hayes L and Martin SJ (2002). CARD games in apoptosis and immunity. Embo Rep. 3: 616-621.

Boyle JP, Mayle S, Parkhouse R and Monie TP (2013). Comparative genomic and sequence analysis provides insight into the molecular functionality of NOD1 and NOD2. Front. Immunol. 4: 317.

Chamaillard M, Girardin SE, Viala J and Philpott DJ (2003). Nods, Nalps and Naip: intracellular regulators of bacterialinduced inflammation. Cell Microbiol. 5: 581-592.

Chen WQ, Xu QQ, Chang MX and Nie P, et al. (2010). Molecular characterization and expression analysis of nuclear oligomerization domain proteins NOD1 and NOD2 in grass carp Ctenopharyngodon idella. Fish Shellfish Immunol. 28: 18-29.

Correa RG, Milutinovic S and Reed JC (2012). Roles of NOD1 (NLRC1) and NOD2 (NLRC2) in innate immunity and inflammatory diseases. Biosci. Rep. 32: 597-608.

Geddes K, Rubino S, Streutker C and Cho JH, et al. (2010). Nod1 and Nod2 regulation of inflammation in the Salmonella colitis model. Infect. Immun. 78: 5107-5115.

Girardin SE, Tournebize R, Mavris M and Page AL, et al. (2001). CARD4/Nod1 mediates NF-kappaB and JNK activation by invasive Shigella flexneri. Embo. Rep. 2: 736-742.

Girardin SE, Boneca IG, Carneiro LA and Antignac A, et al. (2003). Nod1 detects a unique muropeptide from gramnegative bacterial peptidoglycan. Science 300: 1584-1587.

Hayashi T, Nakamura T, Takaoka A (2011). Pattern recognition receptors. Nihon Rinsho Meneki Gakkai Kaishi 34: 329345.

Hruz P, Zinkernagel AS, Jenikova G and Botwin GJ et al. (2009). NOD2 contributes to cutaneous defense against Staphylococcus aureus through alpha-toxin-dependent innate immune activation. Proc. Natl. Acad. Sci. U. S. A. 106: 12873-12878.

Inohara N, Koseki T, Del PL and Hu Y, et al. (1999). Nod1, an Apaf-1-like activator of caspase-9 and nuclear factorkappaB. J. Biol. Chem. 274: 14560-14567.

Inohara N, Koseki T, Lin J and Del PL, et al. (2000). An induced proximity model for NF-kappa B activation in the Nod1/ RICK and RIP signaling pathways. J. Biol. Chem. 275: 27823-27831. 
Inohara, Chamaillard, McDonald C and Nunez G (2005). NOD-LRR proteins: role in host-microbial interactions and inflammatory disease. Annu. Rev. Biochem. 74: 355-383.

Jacobs SR and Damania B (2012). NLRs, inflammasomes, and viral infection. J. Leukoc. Biol. 92: 469-477.

Kawai T and Akira S (2009). The roles of TLRs, RLRs and NLRs in pathogen recognition. Int. Immunol. 21: 317-337.

Keestra AM, Winter MG, Klein-Douwel D and Xavier MN, et al. (2011). A Salmonella virulence factor activates the NOD1/NOD2 signaling pathway. MBio. 2: e00266-11.

Kersse K, Bertrand MJ, Lamkanfi M and Vandenabeele P (2011). NOD-like receptors and the innate immune system: coping with danger, damage and death. Cytokine Growth Factor Rev. 22: 257-276.

King AE, Horne AW, Hombach-Klonisch S and Mason JI, et al. (2009). Differential expression and regulation of nuclear oligomerization domain proteins NOD1 and NOD2 in human endometrium: a potential role in innate immune protection and menstruation. Mol Hum Reprod. 15: 311-9.

Koizumi Y, Toma C, Higa N and Nohara T, et al. (2012). Inflammasome activation via intracellular NLRs triggered by bacterial infection. Cell Microbiol. 14: 149-154.

Koonin EV and Aravind L (2000). The NACHT family - a new group of predicted NTPases implicated in apoptosis and MHC transcription activation. Trends Biochem. Sci. 25: 223-224.

Masumoto J, Yang K, Varambally S and Hasegawa M, et al. (2006). Nod1 acts as an intracellular receptor to stimulate chemokine production and neutrophil recruitment in vivo. J. Exp. Med. 203: 203-213.

Oehlers SH, Flores MV, Hall CJ and Swift S, et al. (2011). The inflammatory bowel disease (IBD) susceptibility genes NOD1 and NOD2 have conserved anti-bacterial roles in zebrafish. Dis. Model. Mech. 4: 832-841.

Opitz B, Puschel A, Beermann W and Hocke AC, et al. (2006). Listeria monocytogenes activated p38 MAPK and induced IL-8 secretion in a nucleotide-binding oligomerization domain 1-dependent manner in endothelial cells. J. Immunol. 176: 484-490.

Pfaffl MW (2001). A new mathematical model for relative quantification in real-time RT-PCR. Nucleic Acids Res. 29 : e45.

Philpott DJ and Girardin SE (2010). Nod-like receptors: sentinels at host membranes. Curr. Opin. Immunol. 22: 428-434.

Robertson SJ and Girardin SE (2013). Nod-like receptors in intestinal host defense: controlling pathogens, the microbiota, or both? Curr. Opin. Gastroenterol. 29: 15-22.

Stern CD (2005). The chick: a great model system becomes even greater. Dev. Cell. 8: 9-17.

Ting JP and Davis BK (2005). CATERPILLER: a novel gene family important in immunity, cell death, and diseases. Annu. Rev. Immunol. 23: 387-414.

Tohno M, Shimazu T, Aso H and Uehara A, et al. (2008). Molecular cloning and functional characterization of porcine nucleotide-binding oligomerization domain-1 (NOD1) recognizing minimum agonists, meso-diaminopimelic acid and meso-lanthionine. Mol. Immunol. 45: 1807-1817.

Travassos LH, Carneiro LA, Girardin SE and Boneca IG, et al. (2005). Nod1 participates in the innate immune response to Pseudomonas aeruginosa. J. Biol. Chem. 280: 36714-36718.

Uematsu S and Akira S (2006). Innate immune recognition of viral infection. Uirusu. 56: 1-8.

Uematsu S and Akira S (2008). Toll-Like receptors (TLRs) and their ligands. Handb. Exp. Pharmacol. 183: 1-20.

Werts C, Girardin SE and Philpott DJ (2006). TIR, CARD and PYRIN: three domains for an antimicrobial triad. Cell Death Differ. 13: 798-815.

Wilmanski JM, Petnicki-Ocwieja T and Kobayashi KS (2008). NLR proteins: integral members of innate immunity and mediators of inflammatory diseases. J. Leukoc. Biol. 83: 13-30.

Zhao Y, Alonso C, Ballester I and Song JH, et al. (2012). Control of NOD2 and Rip2-dependent innate immune activation by GEF-H1. Inflamm. Bowel. Dis. 18: 603-612.

Zimin AV, Delcher AL, Florea L and Kelley DR, et al. (2009). A whole-genome assembly of the domestic cow, Bos taurus. Genome Biol. 10: R42. 